\title{
Interferometer with single-axis robot: design, alignment and performance
}

\section{Alexey Shakun, Aleksandr Santos-Skripko, Oleg Sazonov, Igor Maslov, Nikolay Ignatiev, et al.}

Alexey Shakun, Aleksandr Santos-Skripko, Oleg Sazonov, Igor Maslov, Nikolay Ignatiev, Igor Stupin, Gabriele Arnold, Alexey Grigoriev, Oleg Korablev, "Interferometer with single-axis robot: design, alignment and performance," Proc. SPIE 11128, Infrared Remote Sensing and Instrumentation XXVII, 111280G (9 September 2019); doi: $10.1117 / 12.2535436$

EDent: SPIE Optical Engineering + Applications, 2019, San Diego, California, United States 


\title{
Interferometer with single-axis robot: design, alignment and performance
}

\author{
Alexey Shakun*a ${ }^{*}$, Aleksandr Santos-Skripko ${ }^{\mathrm{a}}$, Oleg Sazonov ${ }^{\mathrm{a}}$, Igor Maslov ${ }^{\mathrm{a}}$, Nikolay Ignatiev ${ }^{\mathrm{a}}$, Igor \\ Stupin $^{\mathrm{a}}$, Gabriele Arnold ${ }^{\mathrm{b}}$, Alexey Grigoriev ${ }^{\mathrm{a}}$, Oleg Korablev ${ }^{\mathrm{a}}$ \\ ${ }^{a}$ Space Research Institute (IKI), 84/32 Profsoyuznaya, 117997 Moscow, Russia; \\ ${ }^{b}$ Deutsches Zentrum für Luft- und Raumfahrt e.V. (DLR), Institute of Planetary Research, \\ Rutherfordstrasse 2, 12489 Berlin, Germany. \\ *avshakun@iki.rssi.ru; phone+74953336245
}

\begin{abstract}
An interferometer is an essential subsystem of the Fourier-transform spectrometer (FTS). We describe an FTS instrument to operate at the surface of Mars based on a Michelson interferometer with hollow retroreflectors. The instrument will operate in two different regimes, observing the solar disc through the atmosphere to measure trace gases, and measuring the thermal emission from the atmosphere to study the planetary boundary layer (PBL). The interferometer has an aperture of $1 \mathrm{inch}$, operates in the spectral range 1.7-17 $\mu \mathrm{m}$, and features low mass and volume $(\leq 1 \mathrm{~kg}$ with all necessary subsystems). Beam splitter and compensator are made of potassium bromide $(\mathrm{KBr})$. A single-axis robot with stepper motor drive provides a linear movement of the retroreflector (the speed stability is about $2 \%$ ) and enables a maximal optical path difference (MOPD) of $15 \mathrm{~cm}$. A reference channel with a distributed-feedback laser diode $(0.76 \mu \mathrm{m})$ and a photodiode ( $\mathrm{Si}$ ) supports the interferogram sampling and the speed stabilization loop. The time to measure one interferogram with a best spectral resolution of about $0.05 \mathrm{~cm}^{-1}$ is $500 \mathrm{~s}$ (the sun tracking regime). In the thermal sounding regime, one measurement of a two-side interferogram (with the spectral resolution of $\sim 1 \mathrm{~cm}^{-1}$ ) takes less than 1 min. Laboratory calibrations with a black body and a laser confirm the design parameters of the instrument.
\end{abstract}

Keywords: Fourier-transform spectrometer, interferometer, IR remote sensing, Martian atmosphere

\section{INTRODUCTION}

The landing platform, with its science payload [1] is a part of the ExoMars mission (joint ESA-Roscosmos project). The launch of ExoMars mission with the landing platform is planned in 2020. The science payload of the surface platform includes several instruments, and many of them target the long term monitoring of processes in the lower Martian atmosphere.

The infrared Fourier-transform spectrometer FAST (Fourier for Atmospheric Species and Temperature) [2] is a part of the landing platform science payload. The instrument is aimed at long term thermal sounding of the Martian atmosphere and retrieval of the aerosol loading (ice and dust). The second science goal is the sensitive detection of minor gaseous species in the atmosphere of Mars, in particular, methane $\left(\mathrm{CH}_{4}\right)$. FAST will measure the total column abundance of minor species by observing the sun from the surface, bridging the contradictory results obtained recently at the surface $[3,4]$ and from the orbit [5].

The design requirements for an FTS to operate at the surface of Mars involve stringent constraints on mass and volume. It required several iterations of modeling and testing to combine high metrological characteristics of the interferometer with its low mass. A crucial parameter is a spectral resolution necessary to achieve a high sensitivity to detect the trace gases. The key solution was to apply a single-axis robot with a geared stepper motor for the displacement of the retroreflector. Such a drive makes the interferometer quite slow, but it allows to achieve a high resolution and a better $\mathrm{S} / \mathrm{N}$ ratio (for pyroelectric detector applied). Also, the design with the linear drive develops in length. After modeling and testing, the required stiffness of the interferometer was achieved, allowing to conserve the optical alignment after expected mechanical loads.

Infrared Remote Sensing and Instrumentation XXVII, edited by Marija Strojnik, Gabriele E. Arnold, Proc. of SPIE Vol. 11128, 111280G · C 2019 SPIE · CCC code: 0277-786X/19/\$18 · doi: 10.1117/12.2535436 
First measurements with the interferometer performed in the laboratory using IR calibration sources confirm the required performances of the instrument. Absorption lines of atmospheric gases and the instrument function was measured for different operating conditions. The radiometric calibration of the interferometer is planned.

This paper is a progress report, providing a somewhat detailed description of the compact (1-inch) interferometer with the single-axis robot designed for the infrared spectral range $(1.7-17 \mu \mathrm{m})$. The basic optical and mechanical properties, as well as the applied technical solutions, are presented. The alignment procedures will be described; the results of the first laboratory calibrations and the spectra retrieved will be discussed.

\section{INTERFEROMETER DESIGN}

The principal design of compact 1-inch interferometer (Michelson scheme) with the single-axis robot was previously discussed $[2,6]$ as a potential subsystem of an FTS for the Martian landing platform. However, the final version of the interferometer (Fig. 1) has several differences and refinements that were implemented after the series of tests, adjustments, and laboratory measurements. A significant contribution to the final design of the interferometer was made during the successful attempt to adapt it to the prescribed mechanical envelope $\left(265 \times 140 \times 75 \mathrm{~mm}^{3}\right)$.

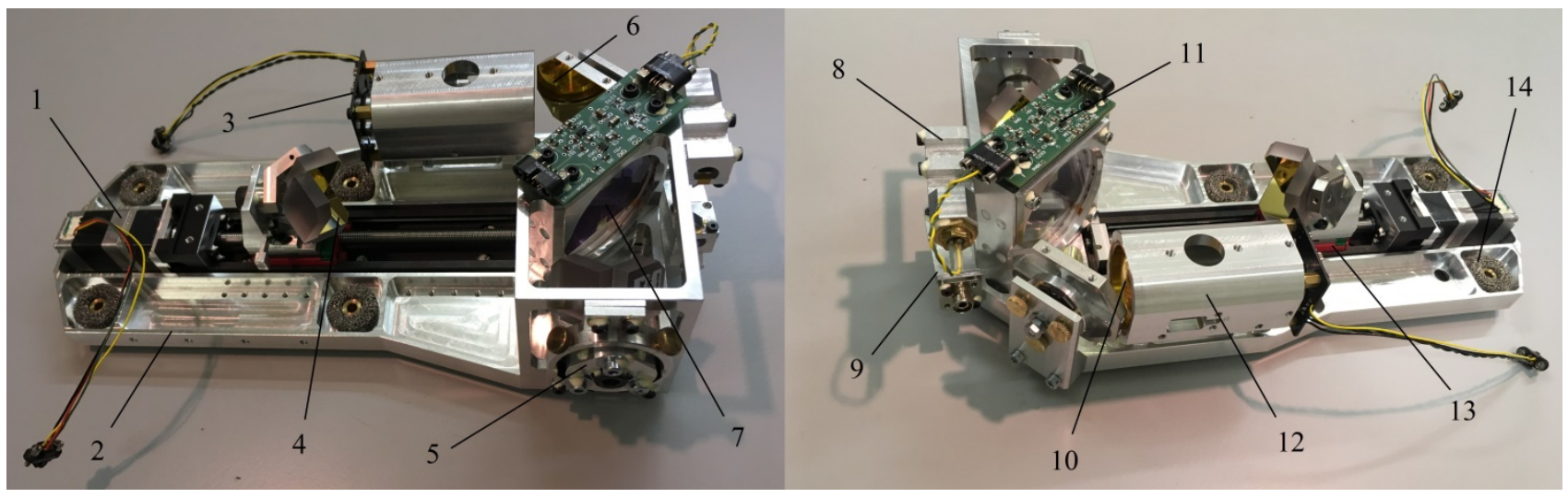

Figure 1. Interferometer with the single-axis robot (different views). (1) geared stepper motor; (2) interferometer base plate; (3) pyrodetector with preamplifier PCB; (4) movable retroreflector; (5) alignment mechanism with the fixed retro reflector; (6) flat mirror (gold plated); (7) beam splitter and compensator pair (KBr); (8) reference channel detector subsystem; (9) reference channel emitter subsystem (DFB laser diode is not connected); (10) spherical (ZnSe) lens; (11) photodiode PCB; (12) telescope; (13) carriage of the single-axis robot; (14) mechanical damper.

The optical scheme of the interferometer [2] has not changed compared to the proposed prototype. The spectral range of the interferometer $(1.7-17 \mu \mathrm{m})$ is defined by the detector inlet window $(\mathrm{Ge})$ with anti-reflectance (AR) coating. The pyroelectric detector (from Leonardo Ltd, UK) with preamplifier PCB is located in the focal plane with the possibility of in-plane and focal adjustment. The diameter of the detector is $3 \mathrm{~mm}$ and the specific detectivity $\mathrm{D}^{*}$ is $1.2 \cdot 10^{9} \mathrm{cmHz}^{1 / 2} \mathrm{~W}^{-}$ ${ }^{1}$ at $10 \mathrm{~Hz}(10 \mathrm{~Hz}$ in the interferogram corresponds to $17 \mu \mathrm{m})$. The spherical lens (ZnSe) with a focal length of $43.5 \mathrm{~mm}$ is used in the telescope of the interferometer. A gold plated flat mirror with an aperture of $28 \mathrm{~mm}$ redirects the interfered beam to the telescope. The retroreflectors (from PLX Inc., USA) have a useful diameter of $25.4 \mathrm{~mm}$, and they are qualified for vacuum applications. The beam splitter and compensator with a diameter $50 \mathrm{~mm}$ and a thickness of $6.5 \mathrm{~mm}$ are made of $\mathrm{KBr}$. The reference channel consists of a visible $(0.76 \mu \mathrm{m})$ distributed feedback (DFB) laser diode (from Eagleyard Photonics, Germany), a detector (Si photodiode made by OSRAM AG, Germany) and a series of optical quartz elements aimed at collimating the laser beam, directing it to the interferometer and subsequently redirecting and focusing it to the detector. The principal design of the reference channel is the same as for our previous interferometers $[7,8]$ but it is implemented more compactly. The beam splitter, the compensator, the flat mirror, and the spherical lens are manufactured by Spectral Systems, LLC (USA). Quartz lenses and prisms of the reference channel are manufactured by LPI (Russia). 
A single-axis robot with geared stepper motor (from Oriental Motor Co., Ltd., Japan) is used to ensure the mechanical support of the optical path difference alteration. The single-axis robot (from HIWIN, Taiwan) has $80 \mathrm{~mm}$ carriage excursion range and 1-mm thread step. A two-phase geared stepper motor (1:50, 200 steps) operating in the micro-step regime (200 micro-steps per 1 step) supports the accuracy of the carriage velocity at a $2 \%$ level (standard deviation). The operation algorithm is based on the PID (proportional-integral-derivative) regulator [9] and uses the signal of the reference channel in the control loop. The optimal PID coefficients can be determined and modified in flight for a specified OPD interval. The implementation of the geared stepper motor with sub-step control makes it possible to achieve an acceptable level of speed stabilization without a two-stage system that has previously been considered [6]. Such simplification makes the interferometer much more suitable for a space application.

The main purpose of the interferometer alignment procedure is to place the image of the stationary retroreflector onto the track of the moving one. A laboratory laser $(\mathrm{HeNe}, 633 \mathrm{~nm})$ and a short-focus lens create a wide-angle beam, which illuminates the interferometer inlet. For an ideally adjusted interferometer, the center of the observed interference picture is stable and does not shift as the retroreflector moves along the rail. If the adjustment is not ideal and the center of the interference picture shifts with the retroreflector displacement, the stationary retroreflector has to be shifted accordingly to stabilize the interference picture. The beam splitter surface is coated with a thin film of germanium $(\mathrm{Ge})$. Thus, only a small fraction of the red laser beam is visible on the control screen. The adjustment has to be performed in a dark room with the screen made of mate film. The stiffness of the interferometer baseplate is vital to conserve the alignment after mechanical impacts. Stiffening ribs have to be applied. The thickness of the base plate $(12 \mathrm{~mm})$ in combination with mechanical dampers is enough to save the adjustment after expected loads (confirmed by qualification test).

To align the interferometer against the telescope a laser beam is pointed to the center of the beam splitter. The divided beams must pass through the centers of the retroreflectors, and, after reversing, pass through the beam splitter and fall onto the center of the flat mirror that redirects the interfering beams to the telescope. The flat mirror can be inclined to align the outgoing ray and the telescope axis. The accuracy of the alignment can be checked by searching the maximum of signal from a calibration IR source.

\section{MEASURED SPECTRA}

The interferometer operations at the surface of Mars include two regimes: the measurement of two-side symmetric interferogram with $0.5 \mathrm{~cm}$ MOPD (for thermal sounding of the atmosphere and retrieval of the aerosol profiles when Sun is not in the instrument field of view), and the measurement of a partially symmetric interferogram with $15 \mathrm{~cm}$ MOPD (to measure of constrain minor atmospheric species while tracking the Sun disk). The duration of the one-side partially symmetric interferogram measurement is $500 \mathrm{~s}$, the measurement of symmetric short interferogram takes about $30 \mathrm{~s}$. The interferogram is digitized at every second zero crossing of the reference signal (sine wave). A short symmetric interferogram contains 13200 points, and a partially symmetric interferogram for high-resolution measurements contains 210500 points. The expected (theoretical) value of the $\mathrm{S} / \mathrm{N}$ ratio is about 1000 at $3 \mu \mathrm{m}$ (single measurement) in the solar tracking regime and about 20 at $15 \mu \mathrm{m}$ (single measurement) in the thermal sounding regime when observing the daytime Martian sky.

A number of laboratory measurements were performed to estimate the interferometer performance. The interferograms of infrared calibration source with different temperatures were measured in a low-resolution regime. Atmospheric features of $\mathrm{CO}_{2}$ (Fig. 2) and water vapor (Fig. 3) are apparent in the obtained spectra. Interferograms of the infrared HeNe laser $(3.39 \mu \mathrm{m})$ were measured in high-resolution regime to estimate the spectral resolution of the interferometer. The measurements were performed for a $12 \mathrm{~cm}$ MOPD (test regime). The retrieved spectrum (Fig. 4) is the instrument response function for the interferometer. The achieved spectral resolution (full width at half maximum, FWHM) is $0.06 \mathrm{~cm}^{-1}$ (not apodised) or $0.09 \mathrm{~cm}^{-1}$ (apodised). It is 1.5 times coarser than the theoretical one. The possible reason for this discrepancy can be associated with the imperfectness of the interferometer alignment. As a next step, radiometric calibration of the interferometer is planned to be performed in a vacuum chamber. 


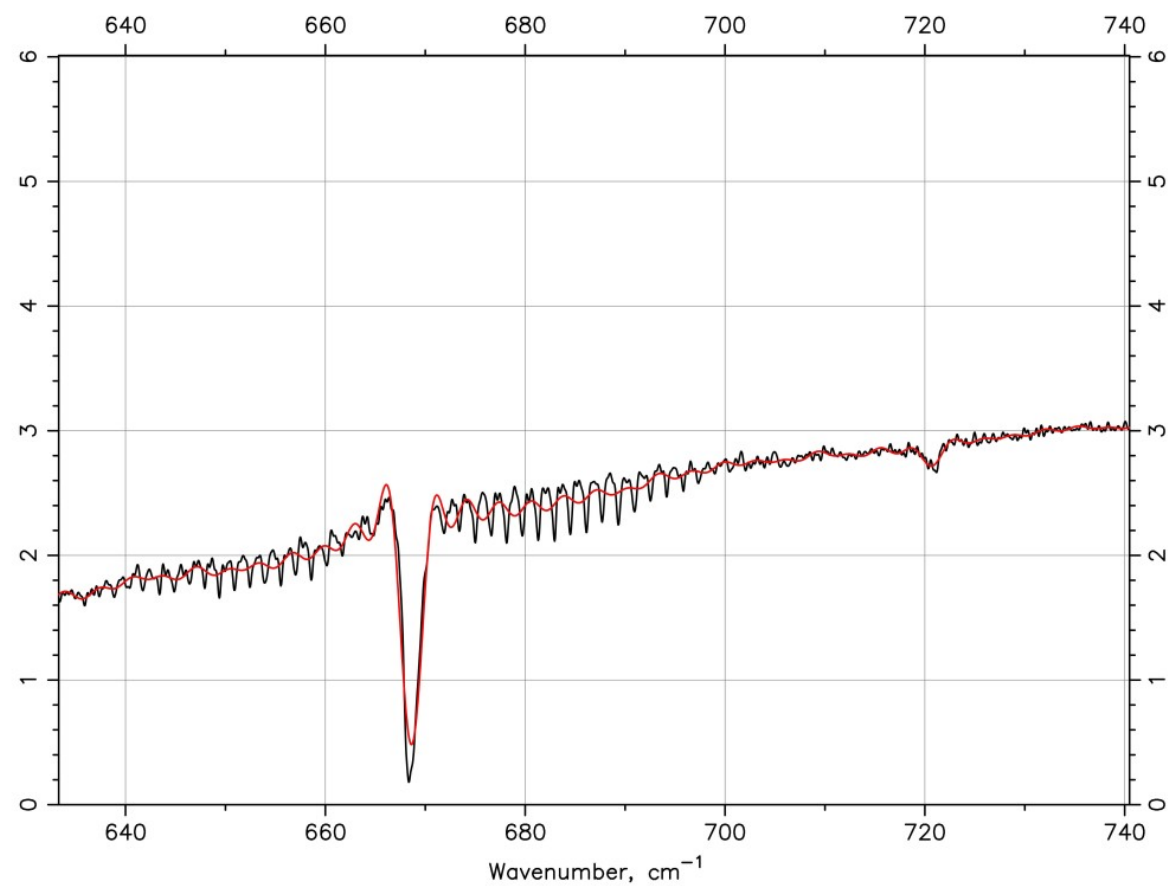

Figure 2. A spectrum measured by the interferometer using the laboratory blackbody calibration source at $973 \mathrm{~K}$. The $\mathrm{CO}_{2}$ $15-\mu \mathrm{m}$ band in the laboratory air is apparent. Black curve- measured spectrum, red curve is the model (preliminary fit; underestimated spectral resolution). Uncalibrated spectra in arbitrary units.

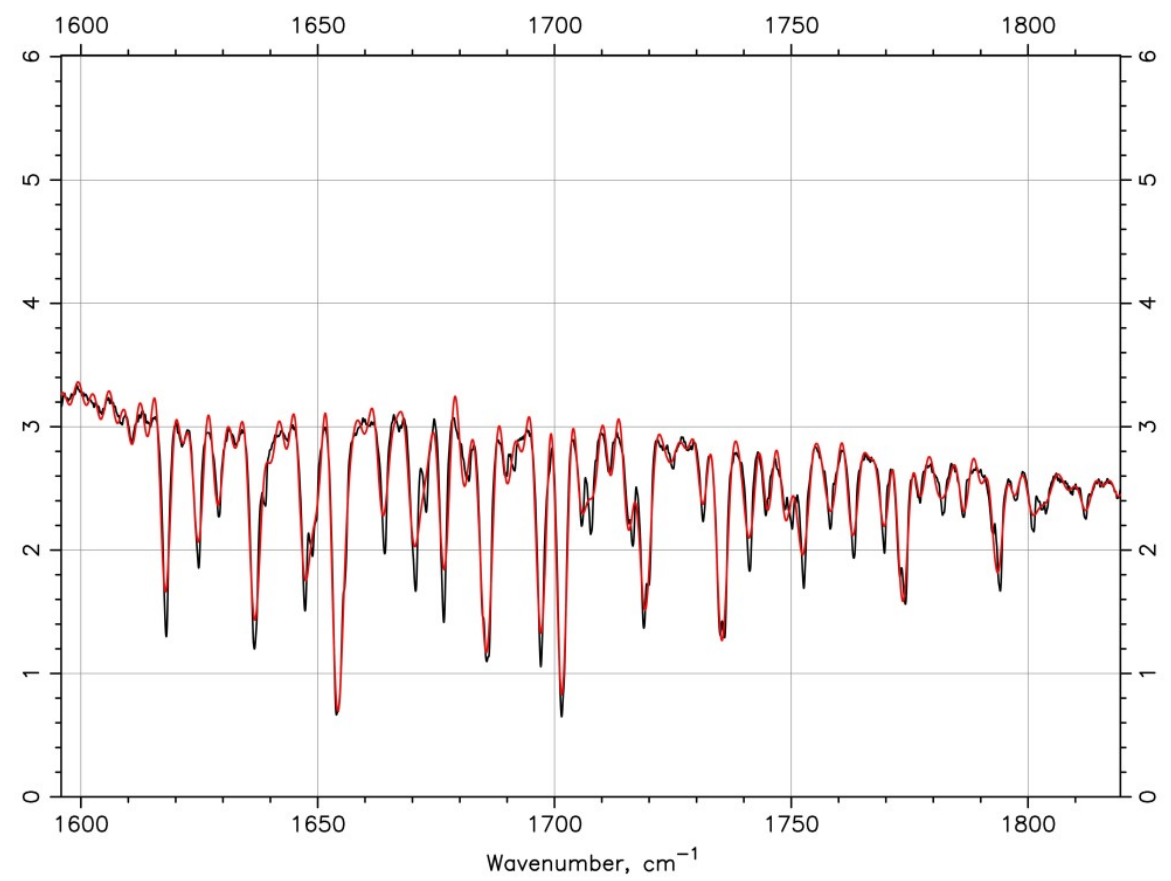

Figure 3. Same as in Fig. 2 showing the $\mathrm{H}_{2} \mathrm{O}$ band at $6 \mu \mathrm{m}$. 


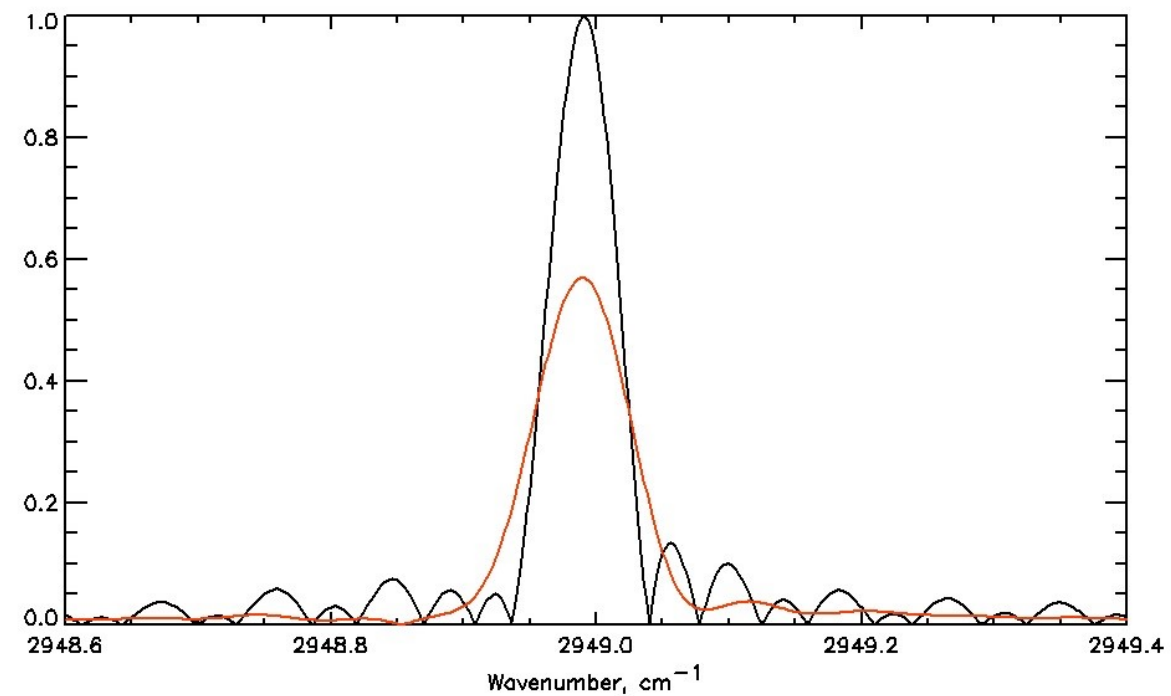

Figure 4. Spectra of He-Ne laser (in arbitrary units) retrieved from partially symmetric interferogram $(\mathrm{MOPD}=12 \mathrm{~cm})$. Black is none apodised and red is apodised (triangular function).

\section{CONCLUSIONS}

A compact 1-inch Michelson interferometer with an integrated single-axis robot is designed for use with the FAST instrument (FTIR spectrometer) for the ExoMars Landing platform. It operates in spectral range 1.7-17 $\mu \mathrm{m}$ and can be used for the thermal sounding of the atmosphere, aerosol retrieval, and measurements of minor atmospheric gases. The use of the geared stepper motor in the micro-stepping regime as a driver for the carriage of a single-axis robot supports the smooth movement of the movable retro reflector with a velocity accuracy of $2 \%$. The operation supported by the PID regulator working in the loop with the reference channel. The MOPD reaches $15 \mathrm{~cm}$. One measurement in highresolution mode takes $500 \mathrm{~s}$. A series of calibration measurements were performed in the laboratory to measure atmospheric gaseous lines $\left(\mathrm{CO}_{2}\right.$ and $\mathrm{H}_{2} \mathrm{O}$ vapor $)$ and estimate the best spectral resolution of the interferometer $\left(0.06 \mathrm{~cm}^{-1}\right.$ for $12 \mathrm{~cm}$ MOPD, not apodised). The radiometric calibration of the interferometer is planned for the near future. The mass of the interferometer is only $1 \mathrm{~kg}$, promising numerous space applications.

\section{ACKNOWLEDGEMENTS}

ExoMars is a space mission by ESA and Roscosmos. The development and fabrication of FAST instrument was funded by Roscosmos. Alexey Shakun, Igor Stupin and Oleg Korablev acknowledge support from Russian Science Foundation (RSF grant 16-12-10453), which enabled to perform experiments, computer modeling and laboratory measurements described in sections 2 and 3.

\section{REFERENCES}

[1] L.M. Zelenyi, O.I. Korablev, D.S. Rodionov, B.S. Novikov, K.I. Marchenkov, O.N. Andreev, E.V. Larionov "Scientific objectives of the scientific equipment of the landing platform of the ExoMars-2020 mission," Solar Syst. Res., 49(7), 509-517 (2015). 
[2] A. Shakun, O. Korablev, B. Moshkin, A. Grigoriev, N. Ignatiev, I. Maslov, O. Sazonov, D. Patsaev, A. Kungurov, A. Santos-Skripko, A. Zharkov, I. Stupin, D. Merzlyakov, V. Makarov, F. Martinovich, Y. Nikolskiy, V. Shashkin "Fourier Transform Spectrometers for remote sensing of planetary atmospheres and surfaces," CEAS Space J., 9(4), 399-409 (2017).

[3] Webster, C. R., P. R. Mahaffy, S. K. Atreya, J. E. Moores, G. J. Flesch, C. Malespin, C. P. McKay, G. Martinez, et al. "Background levels of methane in Mars' atmosphere show strong seasonal variations." Science., 360(6393), 1093-1096 (2018).

[4] Giuranna, M.; Viscardy, S.; Daerden, F.; Neary, L.; Etiope, G.; Oehler, D.; Formisano, V.; Aronica, A.; et al. "Independent confirmation of a methane spike on Mars and a source region east of Gale Crater," Nature Geoscience, 12(5), 326-332 (2019).

[5] Korablev, O.; Vandaele, A.C.; Montmessin, F.; Fedorova, A.A.; Trokhimovskiy, A.; Forget, F.; Lefevre, F.; Daerden, F.; et al. "No detection of methane on Mars from early ExoMars Trace Gas Orbiter observation," Nature, 569(7753), 517-520 (2019).

[6] B.E. Moshkin, I.A. Maslov, O.V. Sazonov, I.G. Stupin "A Fourier-transform spectrometer for operation on Mars," Instrum Exp Tech, 62(2), 247-251 (2019).

[7] O. Korablev, A. Grigoriev, B. Moshkin, D. Patsaev, V. Makarov, S. Maksimenko, K. Grechnev, V. Kotlov, L. Zasova, A. Shakun, A. Fedorova, A. Terentiev, A. Ekonomov, I. Khatuntsev, B. Mayorov, Y. Nikolsky, I. Maslov, A. Gvozdev, F. Montmessin, R. Kuzmin "The AOST miniature Fourier spectrometer for space studies, " J. Opt. Technol. 76(5), 316-322 (2009).

[8] O. Korablev, F. Montmessin, A. Trokhimovskiy, A.A. Fedorova, A.V. Shakun, A.V. Grigoriev, B.E. Moshkin, N.I. Ignatiev, et al., "The Atmospheric Chemistry Suite (ACS) of three spectrometers for the ExoMars 2016 Trace Gas Orbiter," Space Sci Rev, 214:7, doi: 10.1007/s11214-017-0437-6 (2018).

[9] Astrom, K.J., Hagglund T. "Advanced PID control," The Instrumentation, Systems, and Automation Society, North Carolina, 1995. 